\title{
Recourir à l'entre-deux : une proposition pour mieux nommer et décrire la production des espaces informels de Ouagadougou
}

Issa Sory et Abdramane Soura

\section{(2) OpenEdition Journals}

Édition électronique

URL : http://journals.openedition.org/cdg/528

DOI : $10.4000 /$ cdg.528

ISSN : 2107-7266

Éditeur

UMR 245 - CESSMA

Référence électronique

Issa Sory et Abdramane Soura, «Recourir à l'entre-deux : une proposition pour mieux nommer et décrire la production des espaces informels de Ouagadougou », Carnets de géographes [En ligne], 7 | 2014, mis en ligne le 01 décembre 2014, consulté le 30 avril 2019. URL : http:// journals.openedition.org/cdg/528; DOI : 10.4000/cdg.528

\section{(c) (†) $\odot$}

La revue Carnets de géographes est mise à disposition selon les termes de la Licence Creative Commons Attribution - Pas d'Utilisation Commerciale - Pas de Modification 4.0 International. 


\title{
Recourir à l'entre-deux : une proposition pour mieux nommer et décrire la production des espaces informels de Ouagadougou
}

\author{
Issa SORY \\ Institut Supérieur des Sciences de la Population \\ Université de Ouagadougou, Burkina Faso \\ Post-doctorant \\ soryssa@yahoo.fr \\ Abdramane SOURA \\ Institut Supérieur des Sciences de la Population \\ Université de Ouagadougou, Burkina Faso \\ Enseignant-chercheur \\ bassiahi@hotmail.com
}

\section{Résumé}

Le tissu urbain de la capitale burkinabé (Ouagadougou), à l'image de celui d'autres villes au sud du Sahara, est caractérisé par deux grands types de quartiers: quartiers formels et informels. En 2006, environ un quart des Ouagalais vivait dans les quartiers informels. L'une des difficultés majeures à laquelle font face ces territoires urbains est l'absence de services sociaux de base et, de ce fait, ils peuvent être assimilés aux marges spatiales de la ville. A partir d'un état de l'art sur la thématique et les données de l'Observatoire de la Population de Ouagadougou (OPO), cet article met en exergue les jeux d'acteurs à l'origine de la production de ces marges de Ouagadougou, puis il permet de mieux saisir ce qui donne à ces marges leur caractère "transitionnel ». II explicite ensuite les mécanismes de leur marginalisation des politiques publiques en relation avec les services urbains (notamment l'accès à l'eau et l'électricité, et la collecte des déchets), avant de révéler des processus de transition dans l'urbanisation qui invitent à qualifier les quartiers informels de Ouagadougou d'entre-deux, le « entre » semblant finalement plus facile à caractériser que le « deux ".

\section{Abstract}

The urban fabric of the capital city of Burkina Faso (Ouagadougou), like that of other subSaharan cities, is characterized by two main types of districts: formal and informal. In 2006, about a quarter of Ouagalese lived in informal districts. One of the major difficulties faced with by these urban area dwellers is the lack of basic social services. Due to the lack of urban services, these territories may be regarded as spatial margins of the city. Based on the current state of the knowledge on the issue and data from the Observatory of the population of Ouagadougou (OPO), this article highlights the interplay of actors responsible 
for the production of those margins of Ouagadougou in order to better understand the rationale behind their "transitional" nature. Then, it explains the mechanisms of their exclusion from public urban service policies (including access to water and electricity, and garbage collection). Finally, the article spotlights transitional processes in the urbanization development that may be qualified as an "in-between" process; the "between " concept seeming finally easier to characterize than the " two ". 


\section{Introduction}

Estimée à environ 1,75 milliards d'habitants en 1980, la population urbaine a doublé avant 2010 et devrait dépasser le seuil de 4 milliards d'individus à l'horizon 2020 (United Nations, 2011). Dans cette dynamique, nonobstant quelques disparités spatiales, le continent africain connaît l'urbanisation la plus rapide au monde, avec une croissance annuelle moyenne supérieure à 3,4 \% sur les vingt dernières années. En ce qui concerne spécifiquement I'Afrique subsaharienne, quoique sa transition urbaine ait commencé tardivement, le rythme d'urbanisation est tel que plus de la moitié de sa population devrait vivre dans des villes aux alentours de 2040 (United Nation, 2011). Face à ce rythme de croissance et à l'insuffisance de logements qui l'accompagne, des zones d'habitations non cadastrées se sont développées dans plusieurs villes du Sud. Elles abriteraient $62 \%$ de la population urbaine de I'Afrique (NU-Habitat, 2013) et posent des défis importants aux acteurs de la planification et de la gestion urbaine.

Selon les pays, les termes utilisés pour désigner ces territoires urbains sont divers : quartiers irréguliers, quartiers informels ou non lotis, quartiers spontanés, bidonvilles, etc. A bien des égards, ces quartiers sont décrits en opposition au reste de la ville et aux espaces alentours (quartiers réguliers, formels, lotis...) ou comme des espaces en transition vers cette ville dite "légale ", "formelle "... Les termes ne recoupent néanmoins pas les mêmes réalités et méritent d'être bien définis. A partir du cas de Ouagadougou, ce carnet de terrain propose de relire les processus de production de ces quartiers au prisme de l'expression d'entredeux: il s'agit d'interroger sa pertinence, alors qu'elle est peu employée, dans la reconnaissance des particularités et spécificités de la production de ces quartiers selon les contextes nationaux et urbains.

Selon le recensement général de la population et de l'habitation de 2006, 22\% de la population ouagalaise vit dans les zones non cadastrées qui occupent un quart de l'espace urbain (Boyer et al., 2009). Là, ces quartiers sont appelés quartiers informels, irréguliers ou non lotis. En creux, plusieurs critères apparaissent d'emblée inopérants pour expliquer cette distinction. L'évocation des quartiers informels de Ouagadougou renvoie, par exemple, à leur localisation périphérique, alors que certains quartiers formels se trouvent également à la périphérie (Figure 1). De même, l'organisation spatiale du bâti et l'ouverture ou non des routes ne constituent pas des indicateurs fiables de distinction des deux types de territoires urbains ${ }^{1}$. En ce sens, on s'attachera plus spécifiquement à d'autres critères de production des espaces informels, notamment les politiques publiques et les services urbains de base, qui semblent plus percutants d'après l'actualité scientifique et opérationnelle urbaine ${ }^{2}$.

\footnotetext{
${ }^{1}$ La densité du bâti n'y est pas forcément plus élevée que dans le reste de la ville, au contraire, le tissu urbain de Ouagadougou est de plus en plus « lâche » du centre vers la périphérie (Prat, 1996). Cela limite l'usage de la notion de "bidonville " pour les décrire, caractérisés d'après la définition d’ONU-Habitat (2003) par i) le caractère sommaire des matériaux de construction, ii) une forte densité, iii) l'absence de sécurité foncière, iv) le manque de services urbains tels que l'eau potable et v) l'inadéquation du système d'assainissement. Quant aux infrastructures routières, elles se dessinent au fur et à mesure que se construisent les logements définitifs (Deverin-Kouanda, 1993) et expriment donc un état de transition.

${ }^{2}$ L'accès aux services sociaux de base par les résidents de ces quartiers suscite de vifs débats scientifiques (UNHabitat, 2003 ; Dagdeviren et al., 2011 ; Dos Santos, 2007 ; Le Bris, 2002). Parmi les Objectifs du Millénaire pour le Développement (OMD) figurent l'accès de ces citadins aux services urbains (OMD 7), qui participerait à la construction des villes inclusives.
} 
Pour ce faire, la démonstration s'appuie sur des résultats issus de l'Observatoire de Population de Ouagadougou (OPO). Mis en place en 2008 dans deux quartiers formels et trois quartiers informels de la ville, ce dernier constitue une plate-forme d'études et de tests d'interventions visant à nourrir la réflexion des décideurs sur la pauvreté, la santé, l'éducation, l'habitat et l'accès aux services sociaux de base. En juin 2013, l'Observatoire comportait 86251 résidents (définis comme des individus présents depuis plus de 6 mois) dont 46216 dans les quartiers informels. De façon empirique, il s'agit d'analyser comment la complexité des jeux d'acteurs et des politiques publiques à l'œuvre dans la production des quartiers informels de Ouagadougou peut être démêlée par une meilleure appréhension de ce que recoupent l'« entre » et les « deux » si on les qualifie d'espaces de l'entre-deux.

Figure 1 : Tissu urbain de Ouagadougou et quartiers de l'OPO

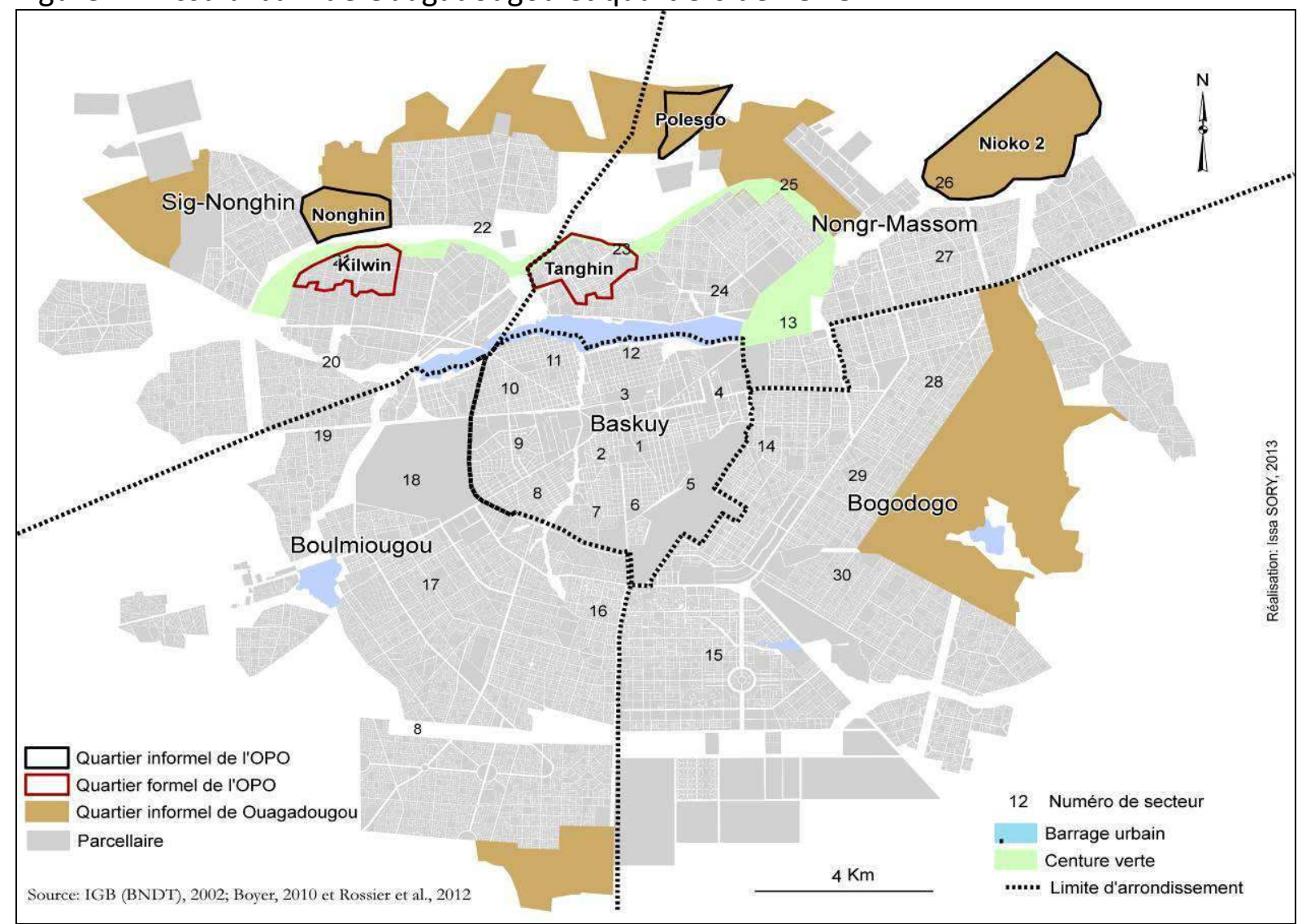

Deux filières de production de l'espace urbain à Ouagadougou : l'entre-deux comme " et " " et "

Dans les pays en développement, les politiques publiques du foncier urbain et leur mise en œuvre par les institutions étatiques se heurtent souvent au régime foncier coutumier préexistant. Comment cette dualité participe-t-elle à la production des quartiers informels à I'intérieur de la ville? 
Une dualité héritée de la colonisation

A Ouagadougou, les quartiers non cadastrés dits "informels" façonnent le tissu urbain depuis l'indépendance du Burkina en 1960. Auparavant, presque toute la ville était " lotie » : "En dehors de quelques enclaves villageoises, il n'existait pas de zones d'habitat spontané à Ouagadougou " (Ouattara, 2005 :191). Il semble même qu'il fallait " contraindre des citadins à occuper les parcelles, l'offre dépassant la demande (ibid.). En 1960, Ouagadougou apparaissait ainsi comme une ville homogène en termes de tenure foncière bien que cela n'empêche certaines inégalités en termes d'équipements.

Cependant, la double structuration du tissu urbain actuel remonte à la colonisation : elle provient de l'introduction de la propriété privée dans la société traditionnelle Mossi par l'administration coloniale ${ }^{3}$ et de l'implication de la chefferie traditionnelle comme acteur de l'attribution du sol urbain ${ }^{4}$. La collusion entre pouvoir colonial et pouvoir traditionnel se traduit par le maintien, de fait, du régime foncier coutumier. Lorsque la Haute-Volta accède à l'indépendance en 1960, se juxtaposent donc dans les localités du Burkina Faso, dont Ouagadougou, deux régimes fonciers (coutumier et moderne), deux acteurs (la chefferie traditionnelle et l'Etat) et deux filières de production du foncier: la filière coutumière, " informelle » ou " populaire » à l'origine de la production des quartiers informels et la filière étatique aménageant la ville légale (Durand-Lasserve, 2004).

\section{Une dualité issue des pratiques des différents acteurs politiques}

Depuis, toutes les politiques urbaines burkinabé ont été émaillées par ces deux filières de production de l'espace urbain, la faiblesse de l'une contribuant au développement de l'autre, et vice-versa. Au-delà du régime juridique, les quartiers dits informels résultent des pratiques des différents acteurs (autorités, résidents et propriétaires terriens) et d'enjeux politiques.

La filière coutumière de production du foncier urbain a dominé pendant la période 19601983: en 1983, 60\% des Ouagalais résidaient dans les quartiers irréguliers qui représentaient $70 \%$ de la superficie de la ville (Jaglin et al., 1992).

En réponse, l'un des objectifs du Conseil National de la Révolution (CNR : 1983-1987), issu du coup d'Etat du 4 août 1983, a été de corriger l'orientation des politiques urbaines appliquées jusque-là. Dans le cadre de sa politique dite " de rupture ", la terre a été nationalisée par la Réorganisation Agraire et Foncière (RAF) ${ }^{5}$. Le domaine foncier national devient la propriété de l'Etat, les titres de propriété antérieurs sont annulés, le titre de jouissance est instauré et les droits coutumiers sont abrogés. Cependant, en milieu urbain, les critères d'attribution des parcelles loties n'aliènent pas, dans les faits, le régime foncier coutumier. Pendant l'attribution des parcelles, l'un de ces critères donne la priorité aux résidents des zones à lotir. Comme pendant la période 1960-1983, les détenteurs des droits coutumiers étaient donc mieux positionnés pour accéder aux droits modernes, d'autant plus que ces aménagements s'accompagnaient rarement de déguerpissement des résidents. La RAF, qui

\footnotetext{
${ }^{3}$ Pendant la colonisation, plusieurs textes juridiques reconnaissaient la propriété privée. Il s'agit entre autres i) de la possibilité d'établir un certificat administratif sur les terres revendiquées et légitimées par la chefferie traditionnelle par le décret du 8 octobre 1925 et ii) de la reconnaissance, par le décret du 28 juillet 1928, du domaine foncier privé (Ouattara, 2005).

${ }^{4}$ Ce qui ne signifie nullement que la chefferie ne gérait pas le foncier avant la colonisation. Au contraire, les chefs de terre, appelés Teng-soba, s'occupaient de l'attribution des terres après avoir effectué les rites traditionnels nécessaires.

${ }^{5}$ Ordonnance n84-050-CNR-PRES du 4 août 1984.
} 
s'apparentait à une loi draconienne envers les propriétaires coutumiers (Bagré, 2000), s'est donc révélée, dans la pratique, comme un pacte entre l'Etat et les chefs coutumiers (Ouédraogo, 1991). Elle n'a pas remis en cause les stratégies qui permettaient aux citadins de se loger dans les quartiers informels et a, au contraire, rendu plus souple les procédures de légalisation des droits coutumiers sur les sols urbains. Pendant la période du CNR, la filière étatique de production de l'espace urbain a donc pris le dessus sur la filière coutumière sans pour autant enrayer les processus coutumiers de production des espaces urbains.

Les relectures de la RAF en $1991^{6}$ et $1996^{7} n^{\prime}$ ont pas supprimé les dispositions accordant la priorité aux résidents des quartiers objets de lotissement ${ }^{8}$. Ces derniers sont prioritaires lors de l'attribution des parcelles et en conséquence, les opérations de lotissement sont toujours précédées d'un recensement des résidents des quartiers informels et entraînent le développement de stratégies pour accéder au sol urbain. Par exemple, des résidents des quartiers formels et les néo-citadins achètent des lopins de terre auprès des chefs coutumiers dans les quartiers irréguliers et y construisent des maisonnettes pour loger, souvent gratuitement, un parent jusqu'à ce que ce dernier soit recensé et que le quartier soit rattaché au foncier moderne urbain.

Devant ces pratiques, une commission interministérielle a été mise en place en 2011 afin d'améliorer la réalisation des opérations de lotissement. Il a été proposé " l'interdiction du recensement dans les zones d'habitat spontané dans le cadre des opérations de lotissement [... et] la destruction des habitations spontanées ${ }^{9}$ " dans les différentes communes. Selon ces recommandations, le recensement des résidents des quartiers informels ne devrait plus se réaliser. Ces quartiers devraient aussi être considérés comme des espaces vacants où les habitations irrégulières sont détruites lors des aménagements urbains. Mais paradoxalement, le 28 juillet 2012, M. Léon Toé, Directeur Général de l'Urbanisme et des Travaux Fonciers (DGUTF), a soutenu que les nouvelles mesures ne visaient pas la destruction des quartiers mais seulement celle des "maisonnettes non habitées ${ }^{10}$ ". En réalité, l'annonce des mesures avait suscité des réactions dans les médias et certains résidents avaient affirmé qu'ils attendaient "de pied ferme " les éventuels destructeurs. Tout porte à croire que les mesures envisagées ne seront pas appliquées et le fait de ne pas pouvoir déguerpir les résidents des zones irrégulières laisse intactes les possibilités de production, de pérennisation et de reproduction de ces quartiers sans rattachement à la ville

\footnotetext{
${ }^{6}$ Kiti n 0328 Ter/FP/PLAN-COOP du 4 juin 1991 portant application de la Réorganisation Agraire et Foncière au Burkina Faso.

7 Décret 97-54/PRES/PM/MEF du 6 février 1997 portant conditions et modalités d'application de la loi sur la Réorganisation Agraire et Foncière au Burkina Faso.

${ }^{8}$ Cf Article 126 du Kiti portant application de la RAF de 1991 repris par l'article 156 du décret portant application de la RAF de 1996: "lorsqu'une opération de lotissement englobe un ou plusieurs villages, il est en outre fait application aux habitants de ce ou ces villages des priorités ci-après: i) demandeurs résidents déguerpis à l'occasion de l'opération de lotissement, ii) demandeurs résidents avec enfants par rapport aux demandeurs sans enfants et aux célibataires [et] iii) demandeurs non-résidents non déguerpis par rapport aux demandeurs non résidents déguerpis. "

${ }^{9}$ Article du ministère de l'Habitat et de l'Urbanisme, « Lotissement au Burkina : Le respect des textes n'est plus négociable », Le Pays, 6 juin 2012, [http://www.lefaso.net/spip.php?article48398, consulté le 26 juillet 2013].

${ }^{10}$ Agence de presse Labor, "Lotissements au Burkina : les non lotis ne seront pas détruits », 29 Juillet 2012, [http://www.laborpresse.net/breves-des-mesures-pour-accroitre-la-production-en-baisse-des-poulets-auburkina/, consulté en ligne le 26 septembre 2013].
} 
légale. L'importance des enjeux, surtout politiques, autour du foncier urbain à Ouagadougou (Sory, 2013) rend inopérantes les mesures de leur éradication.

De "l'informel » à "l'entre-deux »

La reconnaissance tacite du régime foncier coutumier, les stratégies d'accession à la propriété foncière et les pratiques qui leur sont associées rendent problématique la façon de désigner ces quartiers et amènent une multiplication des qualificatifs. Du point de vue de la filière étatique de production de l'espace urbain, ces quartiers sont "informels", car ils résultent de filières coutumières non formelles. Suivant le processus de la légalisation du tissu urbain, ils sont considérés comme des quartiers "non lotis». Les Ouagalais, les autorités et certains auteurs parlent également de "quartiers spontanés » (Jaglin, 1995). Mais ces quartiers ne sont pas construits en un temps relativement court par l'invasion d'espaces suburbains sans aucune forme de négociation et/ou accord préalable avec les propriétaires terriens, comme cela peut se faire dans d'autres villes de l'Amérique Latine et de Turquie (Prat, 1996). Au contraire, ils résultent de filières établies, constituées d'acquéreurs, d'intermédiaires et de propriétaires terriens, aboutissant à l'établissement d'un accord, bien qu'il soit généralement verbal.

Au total, faire appel à "l'entre-deux" pour qualifier les quartiers non cadastrés de Ouagadougou pourrait permettre de mieux rendre compte du double régime juridique et foncier qui les caractérise. La préposition "entre " qualifie ici des espaces "à la fois" soumis au régime étatique et au régime coutumier. Elle sert également à désigner l'état de transition "administrative " de ces quartiers vers la ville légale, du coutumier vers l'étatique : effective à certaines époques, la transition tend à s'installer dans bien des cas. Au-delà, l'expression révèle surtout la complexité des jeux d'acteurs dans la production et la pérennisation du foncier à Ouagadougou et pourrait s'employer dans d'autres espaces urbains burkinabés et africains.

\section{L'entre-deux comme « ni » « ni » facteur de marginalisation}

L'analyse des politiques urbaines en interaction avec le régime foncier coutumier dans la production de l'espace urbain ouagalais a permis de comprendre sa stratification au fil des années en quartiers formels et informels. Mais la limite entre ces deux types de quartiers demeure physiquement floue. Le recours à la statistique révèle la particularité des quartiers informels à travers l'analyse de l'accès aux services de base, notamment les services publics en réseau (eau, électricité) et le système de collecte des déchets. Les quartiers informels se définissent alors par rapport aux quartiers formels de la ville et aux espaces ruraux alentours.

Le propos est ici illustré par une comparaison des quartiers lotis et non lotis de l'OPO avec le milieu rural. Les données sur le milieu rural proviennent de l'Enquête Démographique et de Santé (EDS) de $2010^{11}$, et celles de l'OPO proviennent de la mise à jour réalisée entre mai et

\footnotetext{
${ }^{11}$ L'EDS de 2010 est une enquête nationale représentative des milieux de résidence. Elle a pour objectif de décrire les conditions démographiques et socio-sanitaires de la population. Avec un échantillon de 14424 ménages, elle s'est intéressée aux caractéristiques de l'habitat parmi lesquelles l'accès à l'eau, la présence d'électricité et la gestion des ordures.
} 
novembre 2009. Les ménages de l'OPO sont au nombre de 19167 dont 8018 en quartiers lotis et 11149 en quartiers non lotis.

\section{L'accès à l'eau}

La Figure 2 décrit les sources d'approvisionnement en eau à Ouagadougou selon les zones d'habitation. Dans les quartiers lotis de l'OPO, 47\% des ménages s'approvisionnent en eau potable à travers les branchements individuels à domicile et $36,6 \%$ au niveau des bornes fontaines. A l'inverse, dans les quartiers informels, les bornes fontaines constituent la source principale d'approvisionnement des résidents, utilisées par $70 \%$ des ménages, tandis que $11,7 \%$ des ménages s'approvisionnent au niveau des forages et seuls $2,8 \%$ ont accès au branchement individuel à domicile. En milieu rural, où les forages se substituent aux bornes fontaines, la situation est sensiblement la même que dans les quartiers informels de Ouagadougou et aucun résident ne dispose de branchement individuel. Sur le plan de l'approvisionnement en eau, les quartiers informels se rapprochent donc du milieu rural.

Figure 2 : Source d'approvisionnement en eau selon les zones d'habitation

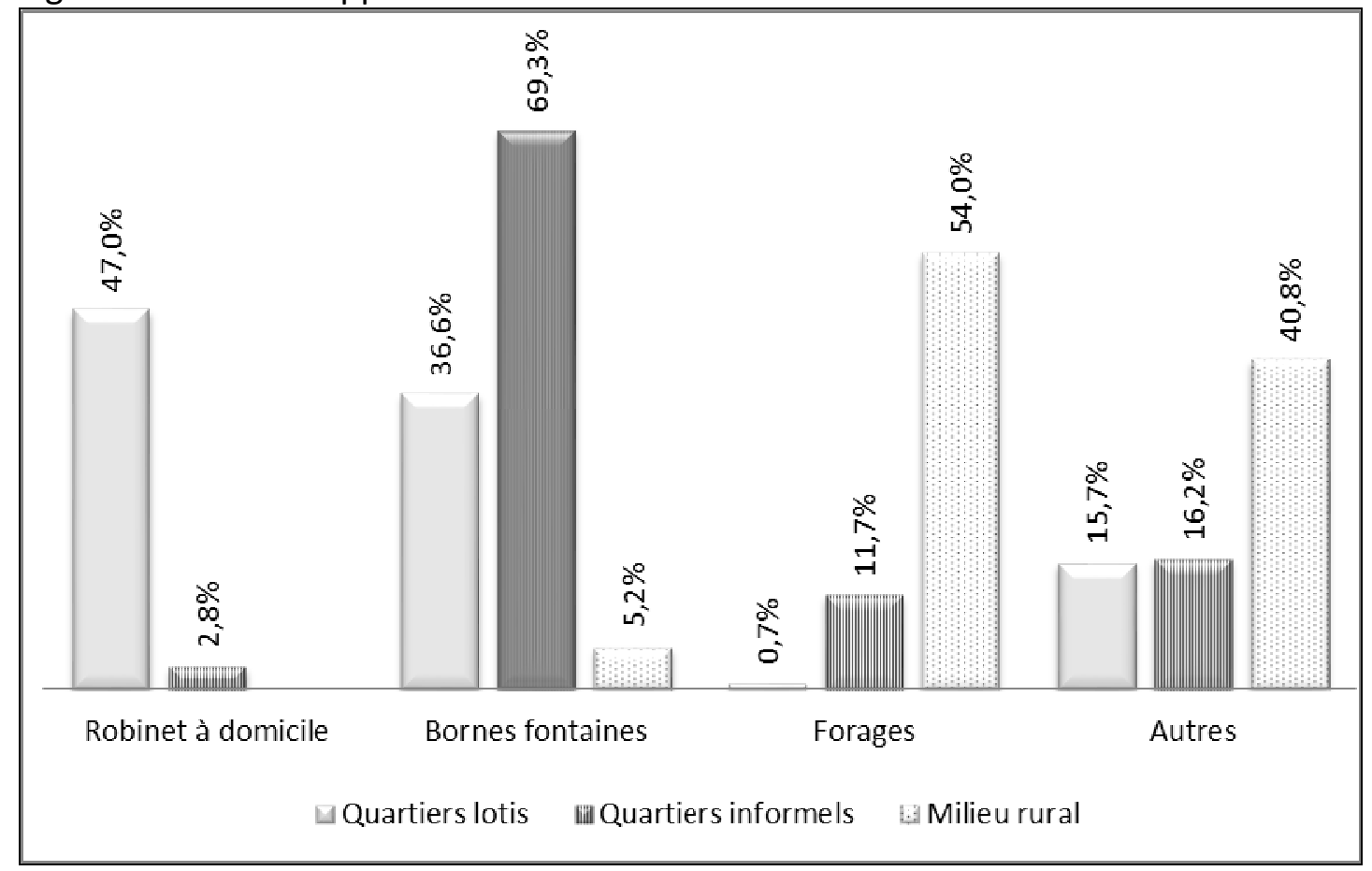

Source : OPO, 2009 et EDS, 2010

Les tendances décrites par les données s'expliquent par une gestion divisée de l'eau entre plusieurs acteurs. Au Burkina Faso, l'Office National de l'Eau et d'Assainissement (ONEA) s'occupe de la desserte d'eau en milieu urbain et est le seul organisme habilité à construire les réseaux d'eau permettant les branchements individuels; tandis que la Direction Générale des Ressources en Eau (DGRE) réalise les forages et les bornes fontaines en milieu rural. L'observation des quartiers d'exercice de I'ONEA indique que les quartiers informels sont exclus du réseau. Lorsqu'il s'agit d'intervenir dans ces espaces, l'ONEA " hésite à aller luimême faire de la gestion de clientèle " (ONEA, 2009 :9) : contrairement aux quartiers lotis et au milieu rural, les quartiers informels semblent moins sécurisés pour l'investissement public et sont considérés comme « dangereux » pour l'offre de service public. 
Aussi, la particularité des quartiers informels est souvent source de conflits de compétence entre I'ONEA et la DGRE. En effet, avant le lotissement de ces quartiers, sous la pression des résidents, la DGRE, en accord avec l'ONEA et les autorités municipales, peut y implanter des infrastructures collectives d'adduction d'eau potable (notamment les forages). Mais en fonction des prévisions des lotissements des quartiers et donc de l'extension du réseau, I'ONEA peut refuser l'intervention de la DGRE. Cette décision contribue à priver ces citadins de ce service, car la réalisation des extensions n'est possible qu'après le lotissement du quartier. Les résidents des quartiers informels, qui sont en situation transitoire "d'attente de rattachement ", perdent ainsi des acquis du milieu rural.

En contrepoint, des bornes fontaines implantées à la lisière de la ville légale desservent les riverains des quartiers informels. II est aussi possible que ces infrastructures soient réalisées par des acteurs privés avec l'autorisation des différentes structures publiques. L'existence de branchements individuels à l'eau courante est ainsi liée à l'existence d'un projet pilote d'adduction d'eau potable dans un des quartiers informels de l'observatoire de la population de Ouagadougou, celui de Nioko 2. II s'agit du projet d'aménagement et de développement des quartiers périphériques de Ouagadougou, financé par l'Agence Française de Développement. Le projet a démarré en 2007 dans cinq quartiers informels de la ville: Bissighin, Zongo, Toukin et Nioko 2 et Bogodogo (Figure 1). La mairie de Ouagadougou constitue le maître d'ouvrage tandis que l'ONEA s'occupe de l'exécution du volet " eau potable et assainissement ". La gestion des bénéficiaires des branchements individuels à l'eau courante a été concédée à de petits opérateurs privés à travers des partenariats publicprivé. Ce partenariat dans le domaine de l'eau et de l'assainissement est de nos jours encouragé avec une conférence nationale tenue du 6 au 8 juin 2013. L'arrivée d'opérateurs privés permet donc le raccordement des quartiers informels au réseau d'eau et tend à limiter les différences entre les milieux urbains informel et formel.

\section{L'accès à l'électricité}

Une démarcation nette s'observe également entre la ville légale et les quartiers informels de Ouagadougou en ce qui concerne l'accès au réseau d'électricité (Figure 3). Alors que plus de la moitié $(55 \%)$ des ménages des quartiers lotis ont accès à l'électricité de la Société Nationale Burkinabé d’Electricité (SONABEL), cette énergie demeure " un luxe " dans les quartiers non lotis où moins de $1 \%$ des ménages y ont accès. Cette proportion semble inférieure à celle observée en milieu rural.

Figure 3 : Les sources d'électricité à Ouagadougou selon les quartiers

\begin{tabular}{|ccc|}
\hline Sources d'énergie & Quartiers formels & Quartiers informels \\
\hline SONABEL & $54,5 \%$ & $0,4 \%$ \\
\hline Batterie & $0,9 \%$ & $5,5 \%$ \\
\hline Lampe & $6,2 \%$ & $13,0 \%$ \\
\hline Torche & $22,8 \%$ & $60,5 \%$ \\
\hline Autres & $15,7 \%$ & $20,6 \%$ \\
\hline
\end{tabular}

Source : OPO, 2009

Les territoires d'intervention de la SONABEL sont les mêmes que ceux de l'Office National de l'Eau et de l'Assainissement (ONEA), les quartiers lotis de la ville. Comme pour le réseau d'eau, des branchements illégaux s'observent sur le réseau d'électricité à la marge des 
quartiers formels, à partir des poteaux électriques. C'est ce qui justifie la présence d'électricité dans des quartiers comme Nioko 2 (Figure 4). En milieu rural, un projet étatique dénommé " Fonds de Développement de l'Electrification (FDE) » est actuellement en cours. Il a été créé en 2003 et érigé en Etablissement public de l'Etat (EPE) en 2010. En une dizaine d'années d'existence (février 2003- décembre 2012), ce fonds a permis l'électrification de quatre-vingt-huit localités, participant ainsi à accroître le taux de couverture du milieu rural en énergie, bien qu'il demeure insuffisant (les prévisions des autorités étaient de couvrir 275 localités en 2012). Ainsi, les quartiers informels apparaissent là encore exclus des politiques publiques de raccordement aux réseaux. Contrairement aux idées reçues concernant une plus grande disponibilité des services de base en milieu urbain qu'en campagne, il n'existe aucune politique d'électrification des quartiers informels de Ouagadougou (Burkina Faso, 2008).

Figure 4 : Branchements temporaires au réseau de la SONABEL à Nioko 2

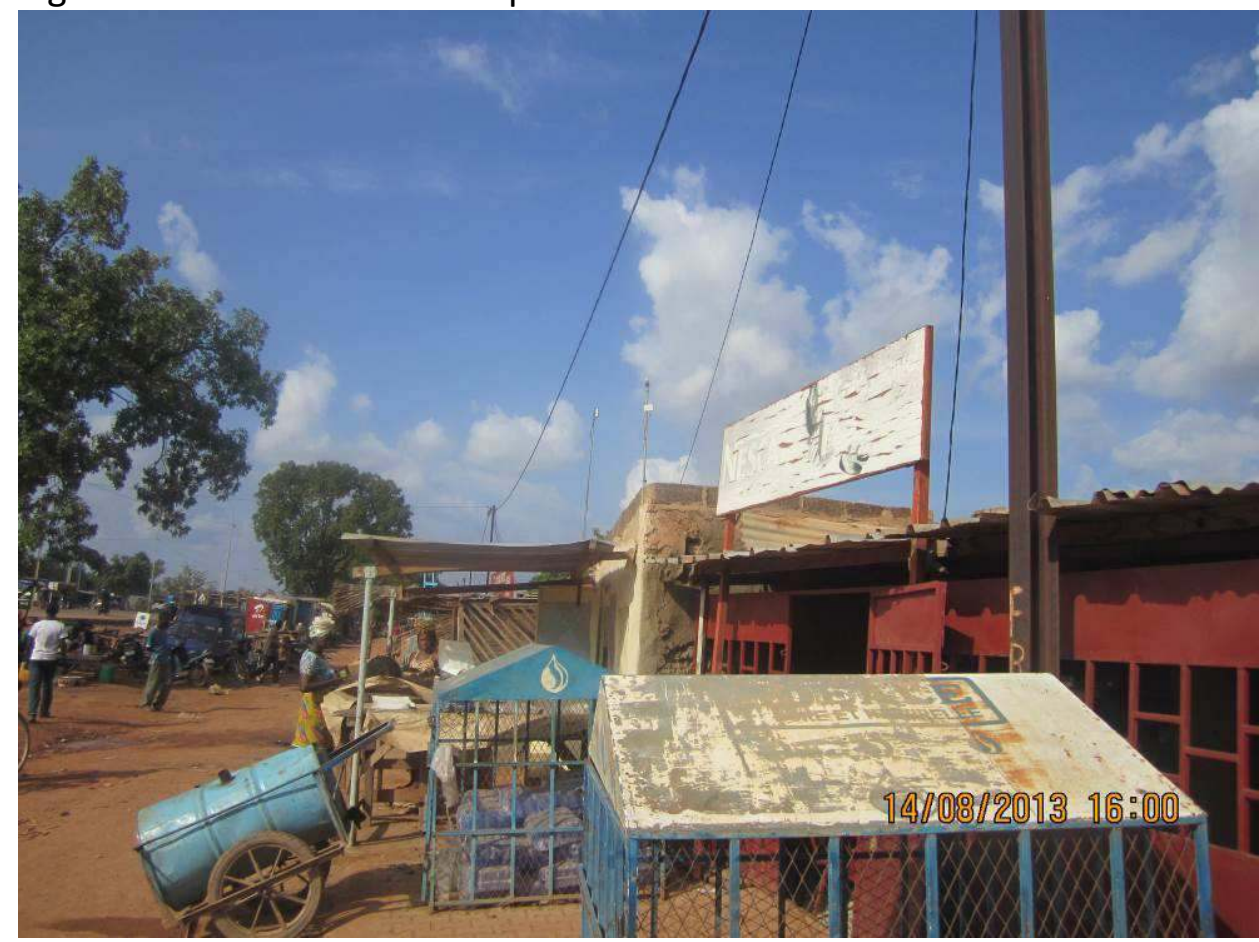

Source : Issa SORY, Août 2013

\section{La collecte des déchets}

L'analyse de la collecte des déchets vient corroborer ces résultats. Au Burkina Faso, l'organisation de la filière des déchets a toujours été du ressort de l'Etat - soit directement, soit à travers des opérateurs privés - de 1960 à la fin des années 1980. Mais avec la décentralisation des années 1990, la pré-collecte, la collecte et l'élimination des déchets relèvent de la compétence des collectivités locales. En conséquence, des acteurs privés ont fait leur apparition dans la filière pour se substituer à l'Office National des Services d'Entretien de Nettoyage et d'embellissement (ONASENE) qui sera " liquidé » en 1996. Cet office, créé en 1988, avait le monopole de la gestion des déchets sur toute l'étendue du territoire national. Pour organiser les pré-collecteurs et collecteurs privés, les autorités municipales, avec le soutien de la Banque mondiale, ont élaboré un Schéma Directeur de Gestion des Déchets (SDGD) en 2000. Avec l'application de ce schéma, à partir de 2005, 
Ouagadougou a été subdivisée en douze territoires de pré-collecte qui ont été attribués, suite à un appel d'offres, aux Petites et Moyennes Entreprises (PME) et Groupements d'Intérêt Economique (GIE). Des infrastructures de collecte tels que les centres de collecte interface entre la pré-collecte et la collecte - et un centre final appelé Centre de Traitement et de valorisation des Déchets (CTVD) ont été construites. Les déchets sont pré-collectés au porte-à-porte et déposés dans les bacs à ordures disposés dans les centres de collecte. Ensuite, la mairie et l'entreprise privée EBTE (Entreprise Burkinabé des Travaux d'Équipements) procède au transport des bacs à ordures des centres de collecte au CTVD. Enfin, dans ce centre, l'enfouissement est l'effectué par une entreprise dénommée "Entreprise de Construction de l'Habitat ".

Les données de l'OPO montrent que $45,30 \%$ des ménages des quartiers lotis font recours aux pré-collecteurs pour l'élimination des ordures ménagères (Figure 5). Ce service est presqu'absent dans les quartiers informels où seulement $0,7 \%$ des ménages éliminent les déchets à travers les pré-collecteurs.

Figure 5 : Modes d'élimination des ordures ménagères

\begin{tabular}{|l|l|l|l|}
\hline Mode d'élimination des déchets & Quartiers formels & Quartiers informels & Milieu rural \\
\hline Services de pré-collecte & $45,3 \%$ & $0,7 \%$ & $0,1 \%$ \\
\hline Nature & $34,3 \%$ & $59,3 \%$ & $62,3 \%$ \\
\hline Rue & $9,9 \%$ & $30,5 \%$ & $2,9 \%$ \\
\hline Autres modes & $10,5 \%$ & $9,5 \%$ & $34,7 \%$ \\
\hline
\end{tabular}

Source : OPO, 2009 et EDS, 2010

L'objectif d'uniformisation des modes de gestion des déchets prôné à travers l'application du SDGD participe au final à la démarcation entre les villes légale et illégale. Comme le révèle l'organisation des infrastructures de collecte des déchets (Figure 6), les pré-collecteurs s'intéressent surtout aux quartiers lotis de Ouagadougou et la quasi-totalité des centres de collecte est localisée dans le tissu urbain légal. Plusieurs facteurs semblent intervenir dans l'exclusion des quartiers informels de la gestion des déchets. Les pré-collecteurs se justifient par l'inaccessibilité de ces quartiers (en traction asine), l'insolvabilité des résidents et l'éloignement des centres de collecte. L'absence d'infrastructures de collecte s'explique par l'illégalité du foncier (qui pourrait conduire à leur destruction lors des opérations de lotissement) et l'absence d'infrastructures routières, empêchant le transport des bacs à ordures (Sory et al., 2012). Les choix techniques du SDGD ${ }^{12}$ sont, de ce fait, inadaptés à la structuration du tissu urbain de Ouagadougou.

\footnotetext{
12 Parmi les choix techniques participant à l'exclusion des quartiers informels figurent le remplacement des bacs à ordures par des espaces construits, les centres de collecte. Les critères du choix des sites de construction de ces centres sont aussi des facteurs de marginalisation de ces quartiers. En effet, le SDGD prévoit que les sites devant accueillir les centres de collecte soient accessibles et disposent d'espace suffisant pour les différentes manœuvres des camions.
} 
Figure 6 : Répartition spatiale des centres de collecte des déchets à Ouagadougou

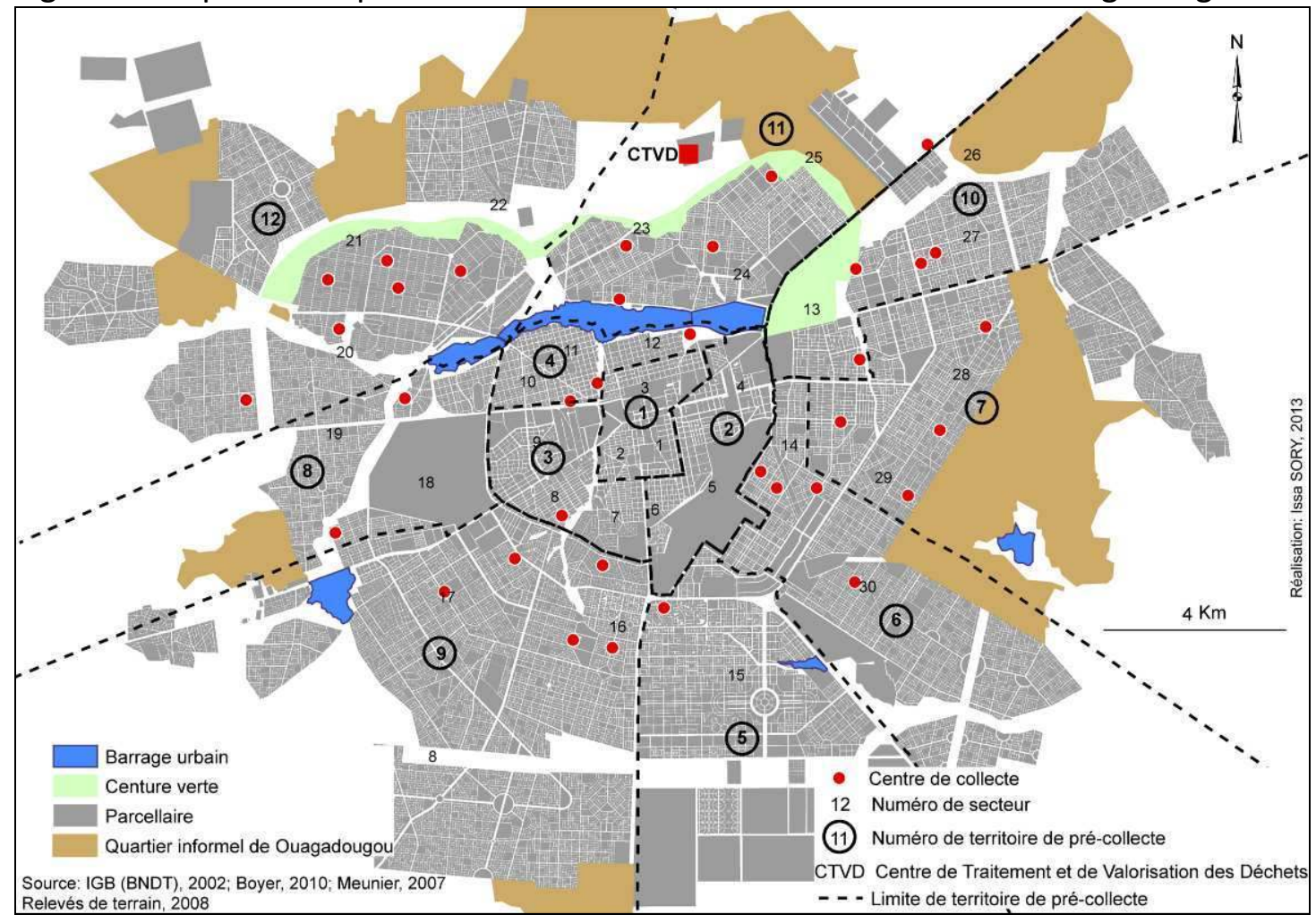

\section{De la marginalisation à "l'entre-deux »}

Comme pour le foncier, après la description du raccordement des quartiers informels de Ouagadougou aux réseaux d'eau, d'électricité et de collecte des déchets, il semble judicieux de recourir à la notion d'entre-deux pour préciser cette informalité. La préposition " entre » est justifiée face à des espaces qui n'appartiennent " ni » au monde urbain légal, " ni » au monde rural et se voient donc écartelés entre les différents acteurs impliqués dans l'accès aux services publics ${ }^{13}$.

La répartition des tâches des différentes structures publiques révèle la démarcation entre quartiers légaux et illégaux et conduit à la marginalisation des quartiers informels. En effet, pour prendre l'exemple de l'eau, il est prouvé que le coût du litre est environ dix fois - voire vingt fois selon Le Bris (2002) - plus élevé pour les ménages qui font recours aux revendeurs $d^{\prime} e^{14}$ que ceux ayant un branchement à domicile (Dos Santos, 2005) : l'eau, du fait de l'exclusion spatiale des quartiers informels du réseau de l'ONEA, est plus chère pour les ménages les moins nantis de Ouagadougou (Dos Santos, 2006), ce qui entraîne leur exclusion sociale. Dépendants finalement soit du processus de lotissement, soit du recours aux acteurs privés, ces quartiers apparaissent comme des espaces flottants du territoire national, " en attente » de raccordement. Les exemples de l'eau et de l'électricité montrent finalement qu'il semble plus avantageux de vivre en milieu rural que dans un quartier

\footnotetext{
${ }^{13}$ A la façon dont Le Bris $(2002: 128)$ le décrit pour l'eau: ces types de territoires « ne relèvent ni de I'hydraulique villageoise ni de l'hydraulique urbaine ".

${ }^{14}$ A Ouagadougou, il existe des revendeurs ambulants qui s'approvisionnent au niveau des bornes fontaines. Le prix du fût de 200 litres qu'ils proposent aux clients est généralement fonction de la distance entre la source d'eau et le ménage et de la saison. Ce prix varie entre 200 et 2500 FCFA.
} 
informel de la capitale burkinabé. D'autres critères, tels l'implantation des établissements scolaires viendraient confirmer cette affirmation ${ }^{15}$. A bien des égards, la transition du milieu rural aux quartiers informels du milieu urbain (par englobement des villages aux périphéries de Ouagadougou sous la croissance urbaine) constitue même une dégradation des conditions de vie des résidents, puisque les habitants passent d'un milieu intégré dans une politique à un espace sans politique, ou en marge des politiques.

\section{Des espaces en attente de légalisation : l'entre-deux comme transition}

Le statut juridique des quartiers informels de Ouagadougou explique leur marginalisation sur le plan des services publics urbains, puisque ces derniers sont réservés en priorité aux quartiers lotis. Les pratiques (coutumières) des acteurs de ces quartiers, tacitement acceptées, contribuent donc à en faire des marges. Cependant, ces pratiques visent également à s'affranchir de ce caractère marginal, avec l'intervention des autorités politiques chargées de lotir ces quartiers.

En effet, comme souligné plus haut, s'installer dans les quartiers informels constitue une étape importante pour l'accès à la ville légale et aux services urbains. Contrairement à certaines marges urbaines (les zones marécageuses d'Abidjan par exemple), les quartiers informels de Ouagadougou ne sont pas physiquement dépréciés. Au contraire, appelés à intégrer la ville légale, ils sont très convoités malgré leur caractère marginal ${ }^{16}$.

Dans cette situation se met donc en place un équilibre entre résidents et autorités politiques, où aucun des deux n'a intérêt à voir le raccordement du quartier aux services de base tant que le lotissement n'a pas été fait.

Du point de vue des autorités politiques, le non raccordement des quartiers informels en services de base (et donc leur marginalisation) constitue une stratégie de contrôle du foncier urbain. En effet, la desserte de ces espaces pourrait rendre leur régularisation moins nécessaire et entraîner une "ruée " de la population vers ces quartiers. Si les conditions de vie (en termes d'accès aux services urbains) sont les mêmes dans les quartiers formels et informels, les habitants auront tout intérêt à s'installer dans les quartiers informels où les coûts du loyer et du foncier sont moins élevés, voire gratuits (Boyer et al., 2009). L'installation des services sans légalisation préalable contribue aussi à renforcer le droit foncier coutumier, contrairement au souhait des autorités publiques. L'absence de politiques publiques ne signifie nullement que ces quartiers échappent aux pouvoirs publics. $\mathrm{Au}$ contraire, la marginalisation des quartiers informels constitue, pour les autorités municipales et étatiques, une des conditions de leur contrôle.

\footnotetext{
${ }^{15}$ Selon l'étude des trois quartiers informels de l'Observatoire de la Population de Ouagadougou, il existe une école publique à Nioko 2 et à Polesgo. Ces deux quartiers ont bénéficié de ces établissements pendant qu'ils étaient toujours des villages. Après qu'ils ont été érigés en quartiers informels, ils n'ont plus connu de construction de nouveaux établissements. Nonghin, l'autre quartier informel, qui n'est pas issu du " grossissement " d'un village mais d'une déportation de Burkinabè rapatriés de la Côte d'Ivoire au début des années 2000, ne dispose d'aucune école publique ni de centre de santé public. Ces différents quartiers informels connaissent ensuite une multiplication d'établissements scolaires privés de qualité douteuse.

${ }^{16}$ Ainsi, sur le plan économique, ces quartiers sont habités par des citadins pauvres comparativement à la moyenne de la ville. Mais ils se vident pendant une partie de la journée au profit de la ville légale où il est plus facile d'obtenir un emploi.
} 
Du point de vue des résidents des quartiers informels, l'accès aux services urbains ne constitue pas une priorité, comme en attestent les revendications des différentes associations de résidents, qui prennent rarement en compte ce volet (Chouli, 2012 ; Sory, 2013). Dos Santos (2007) souligne que pour ces citadins, l'accès à un service essentiel comme l'eau est secondaire par rapport à l'acquisition d'un "chez soi ». Ainsi, les "locataires de concessions, bien que bénéficiant d'un accès à l'eau potable, préféreront s'installer dans des quartiers périphériques dépourvus d'infrastructures de base pour $y$ construire leur logement, sans titre foncier légal, en faisant l'hypothèse que les autorités publiques assureront dans l'avenir le lotissement du quartier, garantie d'insertion durable en milieu urbain " (Baron et al., 2011 :31). Puisque la légalisation du tissu urbain constitue la condition pour accéder aux services, les actions en faveur des lotissements des quartiers informels s'imposent avant toute autre revendication. La recherche de la sécurité foncière, synonyme d'intégration à la ville légale et d'accès aux services sociaux de base, est le facteur significatif de la formation des quartiers informels.

Ces éléments donnent une autre perspective pour qualifier les quartiers informels de Ouagadougou d'espaces de l'entre-deux : la préposition "entre » souligne leur caractère transitoire, " en attente de " lotissement, de légalisation. Cette attente justifie un certain manque d'action et d'initiatives en faveur du raccordement aux services urbains de base et semble donc entraîner l'installation de ces quartiers informels dans cet état transitoire.

\section{Conclusion}

Recourir à la notion "d'espaces de l'entre-deux" pour décrire les quartiers informels de Ouagadougou permet de mieux saisir la complexité des jeux d'acteurs associés à la production de cet espace urbain africain, qu'ils opèrent en matière de juridiction, d'administration et de politiques des territoires, de foncier ou de raccordement aux services publics de base, qu'ils soient publics ou privés, ou qu'ils se réclament du droit coutumier ou moderne. Il est également intéressant de considérer les processus paradoxaux associés à cet entre-deux, qui mettent en continuité (voire dans un rapport de cause à effet) informalisation, marginalisation et légalisation des quartiers non lotis de la capitale burkinabé. L'assimilation des quartiers informels de Ouagadougou à des espaces de l'entredeux permet de mettre en lumière leurs spécificités.

Selon l'angle d'analyse des quartiers et de leurs caractéristiques, l'entre-deux éclaire davantage le « deux " (pour des espaces « et » " et » ou « ni » « ni ») ou le " entre " (comme espaces de "transition vers"). Dans tous les cas, l'étude de ce terrain ouagalais offre un prisme empirique qui rend compte de l'intérêt de la notion pour préciser des concepts relatifs (et/ou inclus dans un couple conceptuel), englobant des réalités diverses, tels ici la marge ou l'informel. Il serait intéressant de déplacer ce prisme à d'autres quartiers informels de villes africaines afin d'en saisir les caractéristiques et spécificités et de tester les récurrences dans les significations du " entre » et du " deux ». Un tel exercice permettrait sans doute de déjouer les catégories spatiales pour accepter davantage de situations spatiales hybrides. 


\section{Bibliographie}

BAGRE A. (2000), Aperçu sur le processus d'urbanisation et la gestion foncière au Burkina Faso : cas de Ouagadougou, Document de conférence AITB, 22 p.

BARON C. et BONNASSIEUX A. (2011) " Les enjeux de l'accès à l'eau en Afrique de l'Ouest: diversité des modes de gouvernance et conflits d'usages ", Mondes en développement, 2011/4, p. 17-32.

BAUTES N. et REGINENSI C. (2008) "La marge dans la métropole de Rio de Janeiro: de l'expression du désordre à la mobilisation de ressources ", Autrepart, 2008/3, p. 149-168.

BOYER F. et DELAUNAY D. (2009), OUAGA 2009: Peuplement de Ouagadougou et Développement urbain, Rapport provisoire, $250 \mathrm{p}$.

BOYER, F. (2010) « Croissance urbaine, statut migratoire et choix résidentiels des Ouagalais : vers une insertion urbaine ségrégée ? ", Revue Tiers Monde, 2010/1, pp. 47-64.

BURKINA FASO (2008), Vision 2020 de l'accès aux services énergétiques modernes, Ouagadougou, MMCE, $89 \mathrm{p}$.

CHOULI L. (2012), Burkina Faso 2011 : chronique d'un mouvement social, Lyon, Tahin Party, $320 \mathrm{p}$.

DAGDEVIREN H. et ROBERTSON S. A. (2011) " Access to Water in the Slums of Sub-Saharan Africa », Development Policy Review, 2011/4, pp. 485-505.

DEVERIN-KOUANDA Y. (1993) «De la fertilité rurale à la nuisance urbaine: les difficiles variations culturelles du Tampuuré (Tas d'ordures) en pays Mossi (Région de OuagadougouBurkina Faso) ", Géographie et cultures, nº, pp. 121-134.

DOS SANTOS S. (2005) " La quête de l'eau en milieu urbain sahélien : I'accès et la perte de l'eau courante à Ouagadougou ", Étude de la population africaine, 19, supplément B, pp. 275-303.

DOS SANTOS S. (2006) " Accès à l'eau et enjeux socio-sanitaires à Ouagadougou - Burkina Faso ", Espace populations sociétés, 2006/2-3, pp. 271-285

DOS SANTOS S. (2007) «L'eau courante à Ouagadougou. Équité et durabilité de l'accès à un service de base ", in Ouedraogo D. et Piché P. (dir.), Dynamique migratoire, insertion urbaine et environnement au Burkina Faso. Au-delà de la houe, Paris, L'Harmattan et Presses Universitaires Ouagadougou, pp. 235-263.

DURAND-LASSERVE A. (2004) "La question foncière dans les villes du Tiers-monde: un bilan ", Economies et Sociétés, Série "Développement, croissance et progrès", $n^{\circ} 42$, pp. 1183-1211.

JAGLIN S. (1995) Gestion urbaine partagée à Ouagadougou : pouvoirs et périphéries (19831991), Paris, Karthala/IRD, 659 p.

JAGLIN S., LE BRIS E., MARIE A., OSMONT A., OUATTARA A., OUEDRAOGO J-P., PIRONAYRAND M., POUSSI M. et SANOU B. (1992), Les enjeux des extensions urbaines à Ouagadougou (Burkina Faso) 1984-1990, Compte rendu de recherche, 365 p.

KIBTONRE G. (1981) Projet pour l'amélioration des conditions de vie et d'habitat des populations à faibles revenus en Haute-Volta, étude d'évaluation exhaustive du projet cinq ans après sa réalisation, $75 \mathrm{p}$.

LE BRIS E. (2002) «Accès à l'eau potable dans les quartiers défavorisés des grandes villes et les petits centres urbains ", in Lévy $M$ (dir.), Comment réduire pauvreté et inégalités : Pour une méthodologie des politiques publiques, Paris, IRD et KARTHALA, pp : 125-138. 
ONEA (Office National de l'Eau et de l'Assainissement) (2009) Projet d'aménagement et de développement des quartiers périphériques de Ouagadougou: Composante Eau Potable et Assainissement, Rapport final d'exécution des prestations du consultant, $60 \mathrm{p}$.

OUATTARA A. (2005) "Gestion foncière et front d'urbanisation au Burkina Faso : le cas de Ouagadougou ", in Coll J-L. et Guibbert J-J., L'aménagement au défi de la décentralisation en Afrique de l'Ouest, Toulouse, Presses Universitaires du Mirail, pp. 181-202.

OUEDRAOGO N. H. (1991), Aménagements urbains et spéculation foncière et immobilière à Ouagadougou de 1960 à nos jours, mémoire de fin de cycle, ENAM, 89 p.

PRAT A. (1996) "Ouagadougou, capitale sahélienne: croissance urbaine et enjeu foncier ", Mappemonde, 1996/1, pp. 18-24.

ROSSIER C., SOURA A., BAYA B., COMPAORE G., DABIRE B., DOS SANTOS S., DUTHE G., GNOUMOU B., KOBIANE J. F., KOUANDA S., LANKOANDE B., LEGRAND T., MBACKE C., MILLOGO R., MONDAIN N., MONTGOMERY M., NIKIEMA A., OUILI I., PISON G., RANDALL S., SANGLI G., SCHOUMAKER B. et ZOURKALEINI Y. (2012) "Profile: The Ouagadougou Health and Demographic surveillance system», International journal of epidemiology, vol. 41, 2012/3, pp. 658-666.

SORY I. (2013) "Ouaga la belle ! " Gestion des déchets solides à Ouagadougou: enjeux politiques, jeux d'acteurs et inégalités environnementales, Thèse de doctorat en Géographie, Université Paris 1 Panthéon-Sorbonne, 355 p.

SORY I. et TALLET B. (2012) « Des choix d'aménagement urbain porteurs d'inégalités sociales et environnementales : La gestion des déchets solides à Ouagadougou (Burkina Faso) ", Flux, 2012/3-4, pp : 79-89.

TRAORE J-M (1986) " Aménagement urbain et pratiques foncières coutumières en HauteVolta ", in Crousse B., Le Bris E., et Le Roy E. (eds.), Espaces disputés en Afrique Noire : pratiques foncières locales, Paris, Karthala, pp : 33-40.

UN-HABITAT (2003), The Challenge of Slums: Global Report on Human Settlements, Nairobi, Earthscan-UN-Habitat, 345 p.

UN-HABITAT (2013), State of the world's cities 2012/2013, New York, Routledge, 185 p.

United Nations (2011) World Urbanization Prospects: The 2011 Revision, [http://esa.un.org/unup/, consulté le 29 juillet 2013]. 